\section{In silico investigation of molecular interactions of Volatile Anesthetics: Effects on phospholipid membranes and subcellular targets}

\author{
Eric A. Zizzi, Marco Cavaglià, \\ Marco A. Deriu, Jack A. Tuszynski \\ PolitoBIOMedLab, Department of \\ Mechanical and Aerospace Engineering, \\ Politecnico di Torino, Turin, Italy
}

\begin{abstract}
The ability of anesthetics to reversibly suppress consciousness must reside in the effects exerted onto specific molecular targets. Interactions between Volatile Anesthetics and the phospholipid membrane as well as intracellular tubulin, were investigated using Computational Molecular Modelling, which showed rapid ligand partitioning inside the membrane and significant effects on the mechanical characteristics thereof, while transient binding locations have been found on the tubulin dimer.
\end{abstract}

\section{Introduction}

Anesthesia, despite being the cornerstone of modern surgery, is to this date a biological puzzle. While scientific efforts still have not managed to pinpoint its exact pharmacological and molecular basis, understanding the effects of Volatile Anesthetics (VAs) on phospholipid membranes and on downstream subcellular molecular targets is a crucial milestone for explaining their complex clinical action. In this context, the interactions between VAs and a model mammalian cell membrane, as well as on human tubulin, the main constituent of microtubules, have been investigated using computational approaches.

\section{Materials and Methods}

a) VA-Membrane simulations: model mammalian membranes composed of POPC, POPS, POPE, PSM and Cholesterol at a ratio of $0.265: 0.085: 0.02: 0.29: 0.34$ (inner leaflet) and 0.1:0.33:0.205: 0.025:0.34 (outer leaflet) ${ }^{1}$ have been assembled using the CHARMM-GUI webserver and simulated for $1 \mu \mathrm{s}$ in the NPT ensemble using the CHARMM $36^{2}$ forcefield in GRO-
MACS 2020.2, at 303.15K and 1 bar. $\mathrm{NaCl}$ counterions have been added to reach the physiological salt concentration of $0.15 \mathrm{M}$. Desflurane (DF) and Methoxyflurane (MF) were added in the solvent at $0 \%$ (control), $12.5 \%, 25 \%$ and $50 \%$ molar ratios with respect to the lipid component. Membrane thickness and area per lipid (APL) were calculated from the simulations. Membrane mechanical stiffness was determined using lipid splay analysis ${ }^{3}$ on the MD simulations.

b) VA-Tubulin simulations: models of human tubulin isotypes $\beta$ VI, $\beta$ IIa and $\beta I V a$ from previous work have been simulated in GROMACS for 100ns using the AMBER ff99SB-ILDN force field 4 following the same NPT protocol as described before. Simulations were performed both without and with DF, MF, Ethylene (ET), and Halothane (HT) in the solvent at fixed 10 mM concentration. 5 The main binding clefts were sampled from MD simulations using residence time analysis and the strength of the interaction in different locations has been quantified using the MM/PBSA method.

\section{Results}

The interaction of DF and MF with model mammalian membranes takes the form of extended spontaneous membrane partitioning of the ligands, taking place in the first 100ns of MD simulations, consistent with their hydrophobic nature. This behavior has effects on both the APL and membrane thickness. More in detail, the inclusion of both DF and MF causes a reduction in membrane thickness from $46.76 \pm 0.29 \AA$ (control) to $45.29 \pm 2.10 \AA$ (MF 50\%) and $45.67 \pm 2.07 \AA$ (DF 50\%), as well as an increase in APL from $42.95 \pm$ $1.40 \AA^{2}$ (control) to $47.53 \pm 2.53 \AA^{2}$ (MF $50 \%$ ) and $47.36 \pm 2.34 \AA^{2}$ (DF 50\%), in a fashion proportional to ligand concentration, as shown in Figure 1 for MF. This is indicative of a progressive contraction of membrane thickness and increased lateral mobility of lipids, as confirmed also by the increasing variability of thickness at higher VA concentrations.

In terms of membrane mechanical bending stiffness, the partitioning of both ligands causes a significant reduction in the monolayer bending modulus from an average of $48.6 \pm 0.6 \mathrm{kT}$ (control) to $35.5 \pm 0.5$ $\mathrm{kT}(\mathrm{MF} 50 \%)$ and $34.6 \pm 0.5 \mathrm{kT}$ (DF 50\%), as highlighted in Figure 2.

The calculated membrane stiffness in control simulations is in good agreement with experimental data, while results in the presence of anesthetics are coherent with previous literature suggesting fluidification
Correspondence: Eric A. Zizzi, PolitoBIOMedLab, Department of Mechanical and Aerospace Engineering, Politecnico di

Torino, Turin, Italy

E-mail: eric.zizzi@polito.it

Key words: Anesthetics; molecular dynamics; membrane; phospholipids; microtubules.

Disclosures: Authors declare no conflict of interest.

Conference presentation: This paper was presented at the Third Centro 3R Annual Meeting - L'era delle 3R: modelli in silico, in vitro e in vivo per promuovere la ricerca traslazionale 30 September - 1 October 2021, Evento online organizzato dal Politecnico di Torino.

Received for publication: 9 July 2021.

Accepted for publication: 7 September 2021.

This work is licensed under a Creative Commons Attribution NonCommercial 4.0 License (CC BY-NC 4.0).

(C) Copyright: the Author(s), 2021

Licensee PAGEPress, Italy

Biomedical Science and Engineering 2021; 4(s1):202 doi:10.4081/bse.2021.202

and increase of membrane compliance upon ligand partitioning, and consistent with the previously found effects on APL and membrane thickness.

Concerning the analysis of the subcellular interaction with tubulin, anesthetics showed distinct interaction patterns with human tubulin dimers, except for ET, which represents the negative control since it is the least potent anesthetic, showing no significant binding. Results confirm the existence of preferential transient binding pockets on the tubulin dimer, and the analysis of average residence time and estimated binding affinity confirmed the lack of interaction with ET: while DF, HT and MT showed comparable affinities between -7 and -14 $\mathrm{kcal} / \mathrm{mol}$, predicted affinities for ET were consistently below $4 \mathrm{kcal} / \mathrm{mol}$, indicative of no significant interaction.

\section{Discussion and Conclusions}

The results suggest that in computational models of mammalian cell membranes, anesthetics rapidly partition in the lipid bilayer, and their presence can significantly alter the overall lipid behavior and membrane mechanics, in the form of a reduction of bending stiffness of more than $10 \mathrm{kT}$. This modification might have profound consequences not only on membrane 


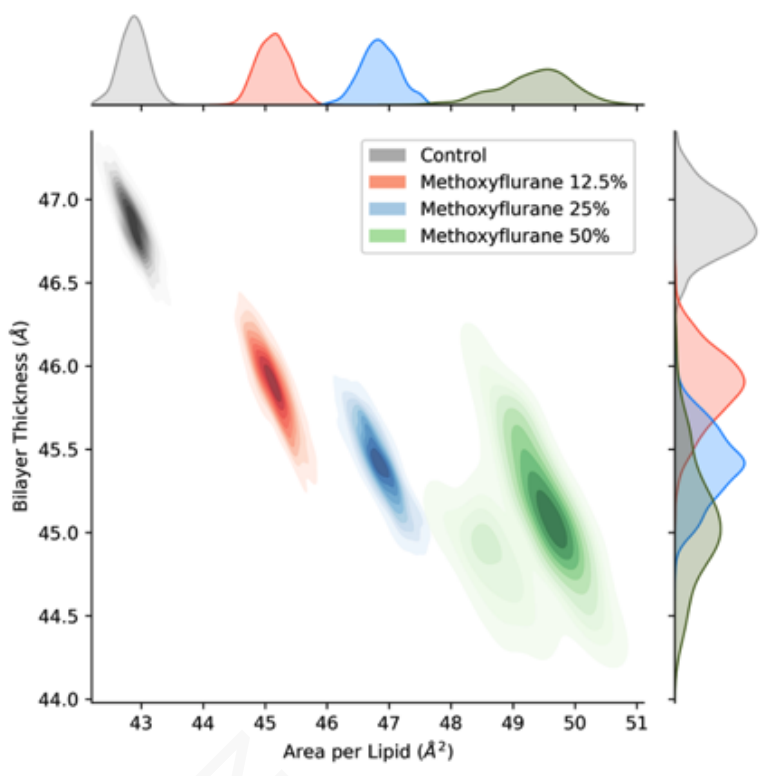

Figure 1. Effect of MF on membrane thickness ( $\mathrm{y}$ axis) and APL ( $\mathrm{x}$ axis) at different concentrations.

behavior itself, but also on the mechanics of channel proteins, currently regarded as the main molecular targets of anesthetics. Inside the cell, on the cytoskeleton, the lack of a unique and stable predicted binding site for volatile anesthetics on tubulin suggests that binding does not follow a lock-and-key paradigm, but can occur repeatedly inside different, energetically favorable clefts, with possible consequences including cross-interactions with MT-targeting chemotherapeutic agents or the exacerbation of side effects of anesthesia, especially in the context of MT-altering neurodegenerative diseases.
A better understanding of the molecular interactions of anesthetics, both in terms of their main mechanism of action and possible off-target interactions, could pave the way for the design of novel anesthetic molecules with improved pharmacology and reduced side effects, through preliminary in silico investigations, reducing unneeded in

\section{References}

1. Shahane G, Ding W, Palaiokostas M, et al. Physical properties of model biologivitro and in vivo trials.

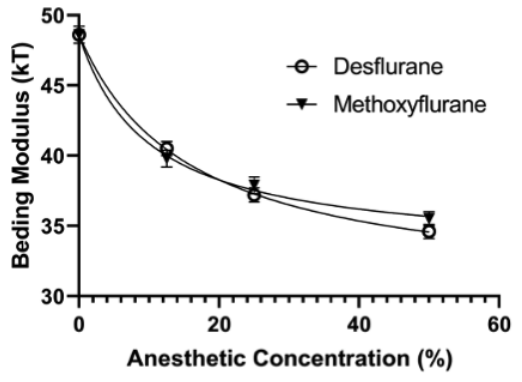

Figure 2. Effect of MF and DF on membrane stiffness as a function of anesthetic concentration.

cal lipid bilayers: insights from all-atom molecular dynamics simulations. J Mol Model 2019;25:76.

2. Klauda JB, Venable RM, Freites JA, et al. Update of the CHARMM all-atom additive force field for lipids: validation on six lipid types. J Phys Chem B. 2010;114:7830-43.

3. Johner N, Harries D, Khelashvili G. Implementation of a methodology for determining elastic properties of lipid assemblies from molecular dynamics simulations. BMC Bioinformatics 2016;17:161.

4. Lindorff-Larsen K, Piana S, Palmo K, et al. Improved side-chain torsion potentials for the Amber ff99SB protein force field. Proteins 2010;78:1950-8.

5. Chau PL. New insights into the molecular mechanisms of general anaesthetics. Br J Pharmacol 2010;161:288-307. 